\title{
A Review of the Application of Carbon Nanotube-based Transistor for Ternary and Quaternary System
}

\author{
Geunho Cho1)
}

\begin{abstract}
The human brain has been considered a very energy-efficient system. With an increase in research and development, understanding the principle of the brain's operation compared with computer systems has significantly improved. In the past decades, most computers operated on binary systems of 0 and 1 ; whereas, the human brain used ternary synaptic weights. Presently, much effort has been made to make hardware architecture similar to the human brain by applying ternary devices that can handle 0 , 1 , and 2 to computer systems. Recently, this approach has attracted considerable attention as it has been known that it becomes hard to improve the performance of an existing semiconductor device, which uses 0 and 1 , with traditional methods (e.g., gate length reduction or structural change). In this paper, we will discuss the various challenges and possibilities to implement ternary (or quaternary) devices and to reduce the number of transistors in circuits with the conventional silicon-based semiconductor devices and next-generation carbon nanotube-based transistors.
\end{abstract}

Keywords: Ternary CMOS, Quaternary Circuit, Carbon Nanotube Transistor, Dual Material Gate Device

\section{Introduction}

In July 2019, [1] indicates that researchers at the Ulsan National Institute of Science and Technology published a paper to confirm the feasibility of ternary semiconductors by implementing ternary metal oxide semiconductors on large-area wafers. These have ultra-low power advantages, high performance, and miniaturization compared to conventional binary semiconductors. As a result, it was confirmed that one semiconductor device could be implemented with three numbers 0,1 , and 2, beyond the binary system of 0 and 1 , considered as the absolute principle of computer operation. Since the human brain, which is known as an energy-efficient system (e.g. operating at lower power (about $20 \mathrm{~W}$ ) and speed (at approximately $10 \mathrm{~Hz}$ ) than conventional semiconductor devices), uses ternary synaptic weights, many researchers have tried to develop ternary semiconductor devices to build foundations for

Received(February 19, 2020), Review Result(1st: March 24, 2020, 2nd: May 16, 2020), Accepted(May 27, 2020)

1) (Professor) 02173 Department of Electronic Engineering, Seokyeong University, 124 Seogyeong-ro, Seongbuk-gu, Seoul, Korea

email: choroot@skuniv.ac.kr 
creating a hardware architecture similar to the human brain[1][2].

With advanced technological development, the size of the semiconductor device and gate length has been reduced to improve the speed of the existing semiconductor and facilitate the functions of a chip. However, when the gate length is smaller than $5 \mathrm{~nm}$, electron transfer can no longer be controlled by the gate voltage due to the quantum tunneling effect. Thus, the gate length $5 \mathrm{~nm}$ is an accepted limitation of the conventional silicon-based transistor. This means that it is hard to increase the speed of a silicon-based transistor any longer by scaling the device. To overcome these limitations, designers have changed the structure of semiconductor devices or used new materials to continuously improve the performance and increase the amount of information that is handled by the semiconductor device. CNTFET(Carbon NanoTube Field Effect Transistor) is widely known as next-generation semiconductor and it has recently been shown that the CNTFET gate length is approximately 1 $\mathrm{nm}$, operating at frequencies of over $100 \mathrm{GHz}$. Previous research has shown that CNTFETs will replace silicon-based semiconductor devices soon. In the present study, we will review the results of the past studies on Ternary CMOS along with the current state of CNTFET development. Moreover, we examine the possibility of CNTFET as the next-generation semiconductor[1-11].

\section{Ternary Circuit}

To implement a ternary circuit, each semiconductor device that constitutes the ternary circuit requires a multi-threshold voltage method in which the individual semiconductor has different threshold voltages. However, a study [1] has created a third voltage state using off-state constant current originating from quantum mechanical band to band tunneling. [Fig. 1] shows the structures of the inverter circuit made of the corresponding semiconductor. [Fig. 2] (a) shows the voltage levels of a conventional CMOS inverter with two voltage levels $(0.0 \mathrm{~V}$ and $1.0 \mathrm{~V})$ and a ternary CMOS with three voltage levels $(0.0 \mathrm{~V}, 0.5 \mathrm{~V}$, and $1.0 \mathrm{~V})$. These three voltage levels represent 0,1 , and 2 in the ternary semiconductor. In the ternary CMOS, more information can be found in one semiconductor device than the conventional CMOS. [Fig. 2] (b) shows the case of converting the incoming binary signal $\left(V_{I N}\right)$ to the ternary signal ( $\left.V_{\text {OUT }}\right)$ by connecting the inverter of [Fig. 1]. 


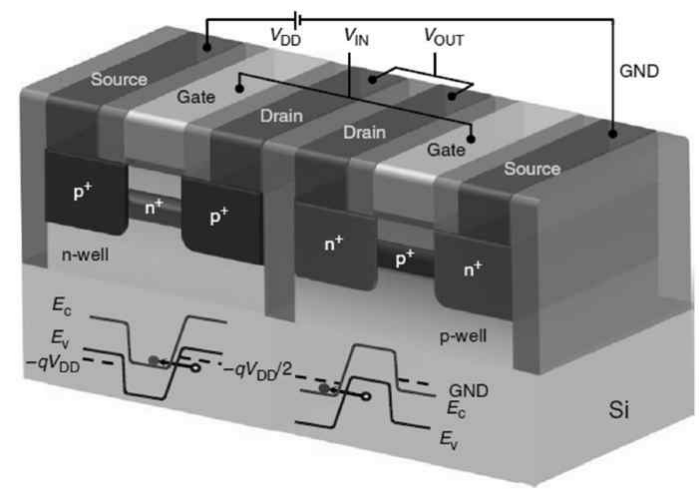

[Fig. 1] Ternary CMOS[1]

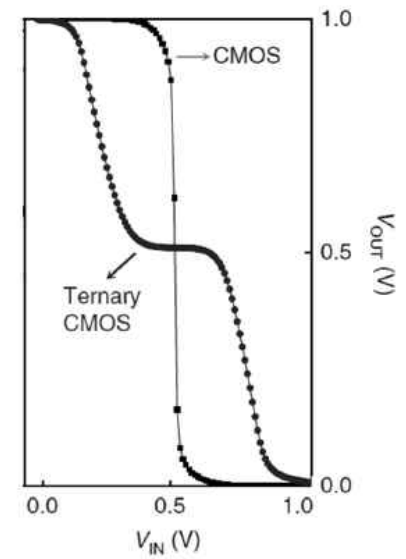

(a)

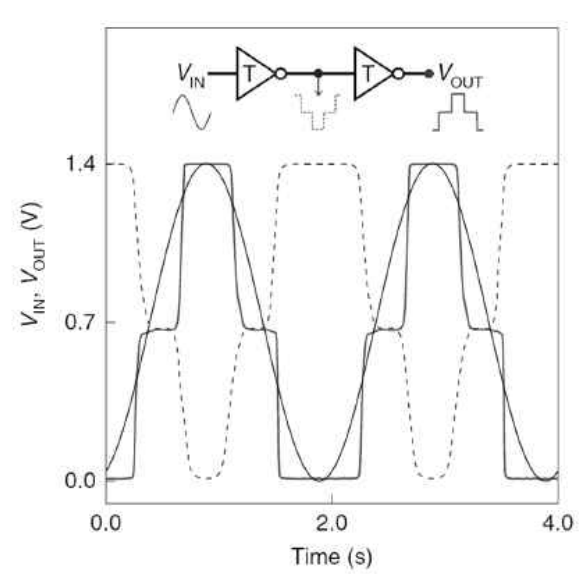

(b)

[Fig. 2] Ternary CMOS[1]

\section{CNTFET}

As shown in [Fig. 3], CNTFET places CNT(Carbon NanoTube), which has a mean free path of approximately 1um, between the source and drain of the semiconductor device. This enables the ballistic or near-ballistic movement of electrons with low bias voltage; so, CNTFET can flow more current with less voltage than conventional MOSFET. The CNT that constitutes the CNTFET comprises of the graphene sheet which is rolled up to form a tube shape. In this case, the diameter of the tube is the main factor that determines the threshold voltage of the CNT channel. Moreover, the CNTFET of different threshold voltages can configure CNTFET 
circuits by placing the CNTs that have different diameters in each CNTFET. In other words, the CNTFETs can make circuits of various threshold voltages operate faster than conventional silicon-based transistors. Additionally, the CNTs also have mechanical flexibility, it makes the CNTFET possible to be implemented on flexible substrates, opening the possibility of their application to wearable devices for the Fourth Industrial Revolution[5-11].

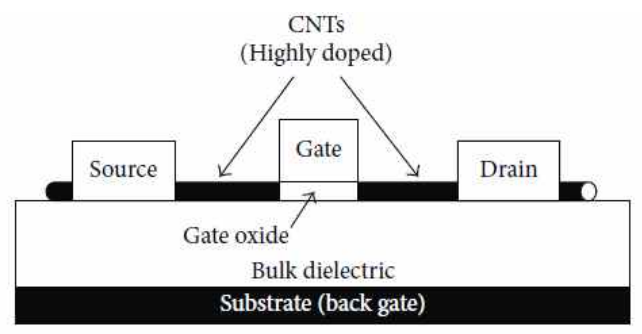

[Fig. 3] CNTFET[6]

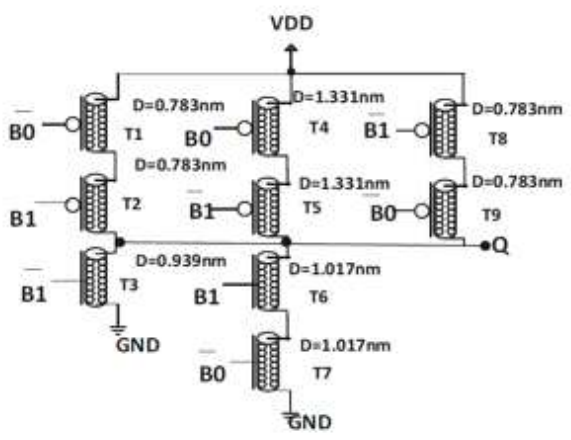

(a)

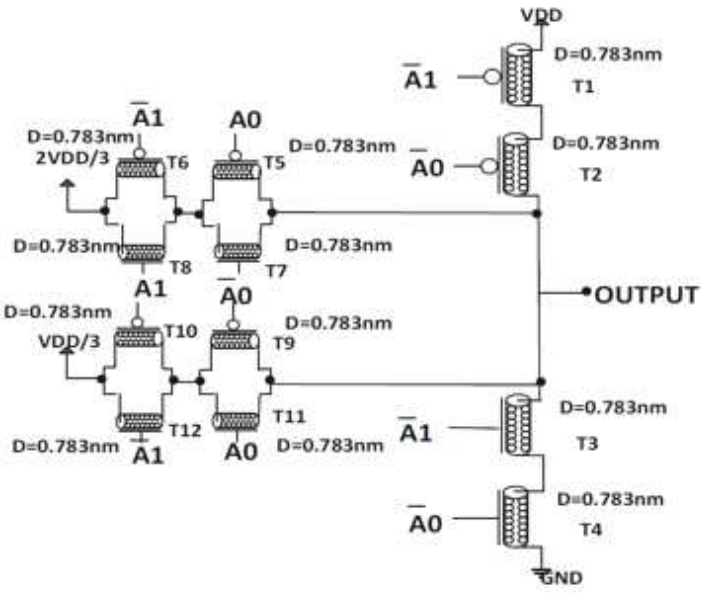

(b)

[Fig. 4] Binary-to-quaternary Converter Circuit[5]

\section{CNTFET Application}

\subsection{Quaternary Circuit}

As discussed in Section 3, a circuit that converts an existing binary signal into a quaternary signal is designed as shown in [Fig. 4][5]. With this, we can handle more information than the ternary semiconductors introduced in Section 2. With two circuits in [Fig. 3], the cylinders 
located in each transistor represent the CNTs as explained in Section 3. The value of ' $\mathrm{D}$ ' next to each CNT represents the 'Diameter' of each CNT, and each CNTFET shown in [Fig. 3] has a different threshold voltage. The binary-to-quaternary circuit introduced by [5] is presented in two main categories as shown in [Fig. 4]. One has one power supply voltage and nine CNTFETs as shown in [Fig. 4] (a) and another has three power supply voltages and 12 CNTFETs as shown in [Fig. 4] (b).
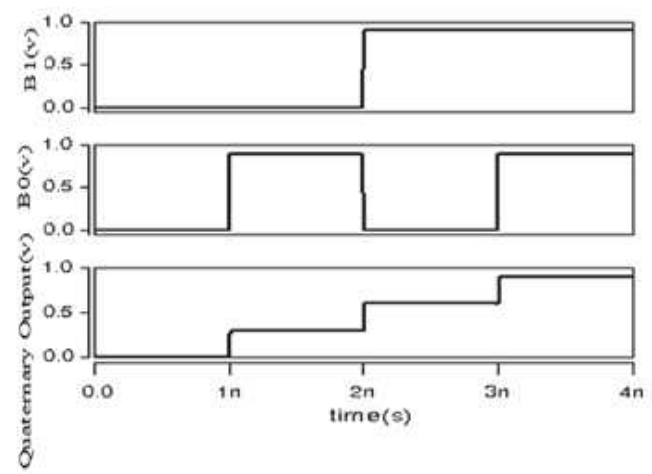

(a)
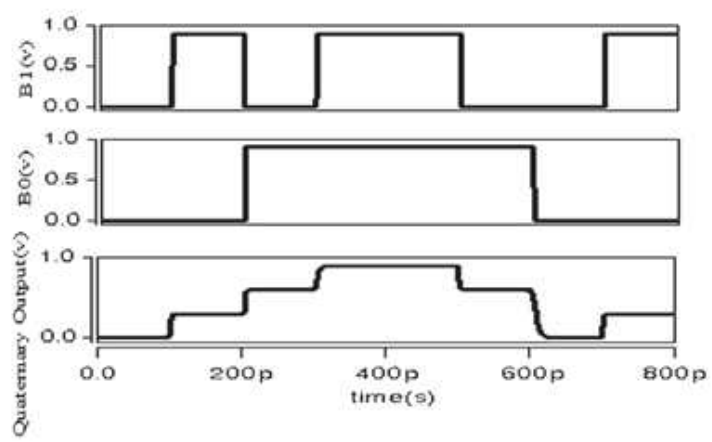

(b)

[Fig. 5] Input and Output Waveforms of Binary-to-quaternary Converter Circuit[5]

When the simulation results of the two circuits are examined, the four voltage levels $(0.0 \mathrm{~V}$, $0.3 \mathrm{~V}, 0.6 \mathrm{~V}$ and $0.9 \mathrm{~V}$ ) are shown in [Fig. 5] (a) and [Fig. 5] (b) respectively. These four voltage levels represent $0,1,2$, and 3 , respectively in the quaternary system. This indicates that they can represent more information than conventional binary or ternary circuits. For performance comparison, the Ternary CMOS introduced in Chapter 2 shows that it takes about 0.4 seconds to change from one voltage level to the next voltage level, whereas quaternary circuits using CNTFETs shown in [Fig. 5] (a) and (b), it takes about 1 ns and 0.1 ns respectively. When considering [Fig. 2] (b) is the measured values and [Fig. 5] is the simulated values, a study [1] explains that a direct pad probing measurement causes a delay of several hundred milliseconds; so, simple numerical comparisons between two values can be a bit unfair. But, since there is a big difference in the unit of measurement of speed (approximately $10^{12}$ ), it can be a meaningful values for reference to estimate the performance difference between a conventional silicon-based transistor and a CNT-based transistor. As Ternary CMOS has successfully implemented on large-area wafer, if the ternary or quaternary circuits made of CNTFETs have also succeeded in enhancing wafer implementation, the two measurements can 
be a good comparison. Currently, CNTFET circuit wafer implementation has succeeded in implementing the 16-bit processor and SRAM based on a binary system. A further study is required to compare fairly the performance values of the two semiconductor devices[9][10].

\subsection{Dual-material Gate Device}

The use of CNTFETs in Section 4.1 has shown the possibility of operating circuits that can represent more information and faster than Ternary CMOS. However, CNTFETs has some disadvantages because it requires more semiconductor devices than Ternary CMOS. To overcome this drawback, it is worth investigating Dual Material Gate Devices using CNTFETs [11], which divide the gate of a semiconductor device in half as shown in [Fig. 6] (a). This consists of a low work function metal $\mathrm{Al}$ (work function $=4.08 \mathrm{eV}$ ) and a high work function metal Pd (work function $=5.2 \mathrm{eV}$ ), configurable functions that enable CNTFETs to operate as transistors or diodes[11]. Among the various functions presented by [11], the notable part is the implementation of the voltage adder and the OR gate in one transistor. Recalling that two circuits require at least four semiconductor devices as shown in [12][13], we can know that, when we use CNTFET, the two functions can be selected and implemented in a single semiconductor device. This means that more functions can be implemented in fewer semiconductor devices in a semiconductor chip with CNTFET soon. Among the waveforms shown in [Fig. 6] (c), the first waveform shows that the voltage adder or the OR gate function is selected in one semiconductor device according to the user's needs. The second and third waveforms show two input waveforms, and the last waveform shows that the two input waveforms output the required waveforms depending on the selected function. Another well-known advantage of CNTs is that CNTs have mechanical flexibility; the CNTFET introduced in Chapter 2 can be implemented on a flexible substrate as shown in [Fig. 6] (a), applicable to human skin as shown in [Fig. 6] (b).

\section{Conclusion}

In the last few years, silicon-based transistors have generally increased their performance by reducing the size of transistors. As the physical limits of the transistors make it difficult to control the movement of electrons, there is a growing interest in designing new materials to overcome the speed limits of transistors and systems to process more information. In these circumstances, it is expected that, in the near future, carbon nanotube-based transistors can 


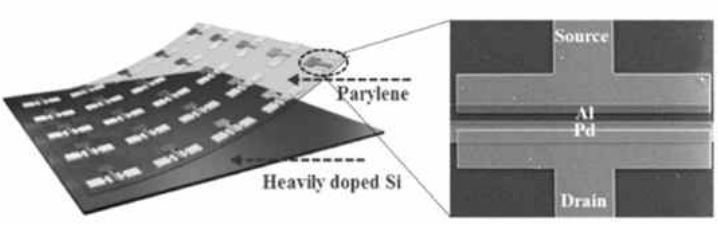

(a)

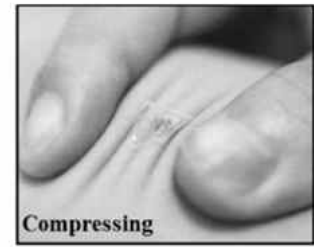

(b)

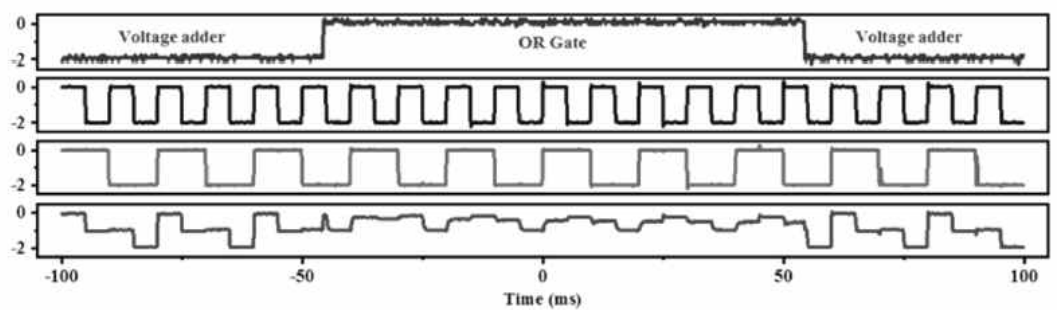

(c)

[Fig. 6] Carbon Nanotube Dual-material Gate Device[11]

\section{Acknowledgement}

This Research was supported by Seokyeong University in 2020.

\section{References}

[1] Jae Won Jeong, Young-Eun Choi, Woo-Seok Kim, Jee-Ho Park, Sunmean Kim, Sunhae Shin, Kyuho Lee, Jiwon Chang, Seong-Jin Kim, Kyung Rok Kim, Tunnelling-based ternary metal-oxide-semiconductor technology, Nature Electronics, (2019), Vol.2, pp.307-312, DOI: https://doi.org/10.1038/s41928-019-0272-8

[2] Steven K. Esser, Paul A. Merolla, John V. Arthur, Andrew S. Cassidy, Rathinakumar Appuswamy, Alexander Andreopoulos, David J. Berg, Jeffrey L. McKinstry, Timothy Melano, Davis R. Barch, Carmelo di Nolfo, Pallab Datta, Arnon Amir, Brian Taba, Myron D. Flickner, and Dharmendra S. Modha, Convolutional networks for fast, energy-efficient neuromorphic computing, Proceedings of the National Academy of Sciences of the United States of America, (2016), Vol.113, No.41, pp.11441-11446.

[3] Sujay B. Desai, Surabhi R. Madhvapathy, Angada B. Sachid, Juan Pablo Llinas, Qingxiao Wang, Geun Ho Ahn, Gregory Pitner, Moon J. Kim, Jeffrey Bokor, Chenming Hu, H. S. Philip Wong, Ali Javey, $\mathrm{MoS}_{2}$ transistors with 1-nanometer gate lengths, Science, (2016), Vol.354, pp.99-102.

[4] Zhong, Donglai, Huiwen Shi, Li Ding, Chenyi Zhao, Jingxia Liu, Jianshuo Zhou, Zhiyong Zhang, Lian-Mao 
Peng, Carbon Nanotube Film-Based Radio-Frequency Transistors with Maximum Oscillation Frequency above $100 \mathrm{GHz}$, ACS Applied Materials \& Interfaces, (2019), pp.42496-42503.

[5] Ghelichkhan, Maryam, Seied Ali Hosseini, Seyyed Hossein Pishgar Komleh, Multi-Digit Binary-to-Quaternary and Quaternary-to-Binary Converters and Their Applications in Nanoelectronics, Circuits, Systems, and Signal Processing, (2019), Vol.39, pp.1920-1942, DOI: https://doi.org/10.1007/s00034-019-01235-6

[6] Shimaa Ibrahim Sayed, Mostafa Mamdouh Abutaleb, Zaki Bassuoni Nossair, Optimization of CNFET Parameters for High Performance Digital Circuits, Advances in Materials Science and Engineering, (2016), Vol.2016, pp.1-9, DOI: https://doi.org/10.1155/2016/6303725

[7] Editorial, 20 years of nanotube transistors, Nature Electron, (2018), Vol.1, No.3, p.149.

[8] Geunho Cho, A Study on the Application and Current Status of Carbon Nanotube-based Transistor, Asia-pacific Journal of Multimedia Services Convergent with Art, Humanities, and Sociology, (2019), Vol.9, No.11, pp.1145-1153.

[9] Gage Hills, Christian Lau, Andrew Wright, Samuel Fuller, Mindy D. Bishop, Tathagata Srimani, Pritpal Kanhaiya, Rebecca Ho, Aya Amer, Yosi Stein, Denis Murphy, Arvind, Anantha Chandrakasan, and Max M. Shulaker, Modern Microprocessor Built from Complementary Carbon Nanotube Transistors, Nature, (2019), Vol.572, No.7771, pp.595-602.

[10] Kanhaiya, P. S., C. Lau, G. Hills, M. D. Bishop, M. M. Shulaker, Carbon Nanotube-Based CMOS SRAM: 1 Kbit 6T SRAM Arrays and 10T SRAM Cells, IEEE Transactions on Electron Devices, (2019), Vol.66, No.12, pp.5375-5380.

[11] Xiang, Li, Fan Xia, Wanlin Jin, Xiangwen Zeng, Fang Liu, Xuelei Liang, Youfan Hu, Carbon Nanotube Dual-Material Gate Devices for Flexible Configurable Multifunctional Electronics, Carbon, (2020), Vol.161, pp.656-664, https://doi.org/10.1016/j.carbon.2020.01.084

[12] M. Al-Nsour, H. S. Abdel-Aty-Zohdy, Simple low power analogue MOS voltage adder, Electronics Letters, (1999), Vol.35, No.7, pp.552-553.

[13] Jan M. Rabaey, Anantha Chandrakasan, Borivoje Nikolic, Digital Integrated Circuits, USA: Prentice Hall, (2003) 